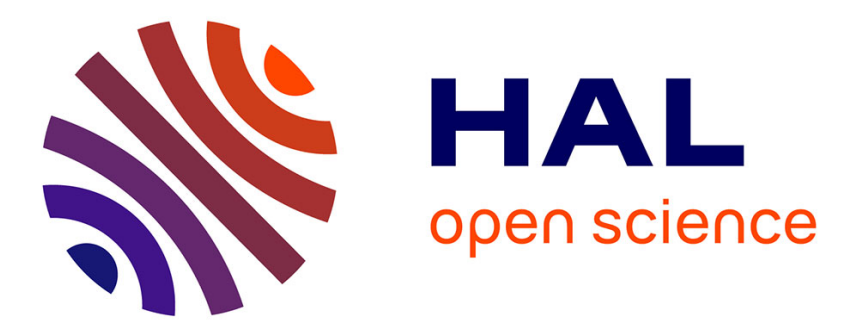

\title{
L'immigration en France des enfants nés à l'étranger
}

Xavier Thierry, Tatiana Eremenko

\section{To cite this version:}

Xavier Thierry, Tatiana Eremenko. L'immigration en France des enfants nés à l'étranger. Recherches familiales, 2009, À l'interaction du géographique et du social: la famille (im)mobile, 6, pp.43-54. 10.3917/rf.006.0043 . hal-03472922

\section{HAL Id: hal-03472922 \\ https://hal.science/hal-03472922}

Submitted on 12 Jan 2022

HAL is a multi-disciplinary open access archive for the deposit and dissemination of scientific research documents, whether they are published or not. The documents may come from teaching and research institutions in France or abroad, or from public or private research centers.
L'archive ouverte pluridisciplinaire HAL, est destinée au dépôt et à la diffusion de documents scientifiques de niveau recherche, publiés ou non, émanant des établissements d'enseignement et de recherche français ou étrangers, des laboratoires publics ou privés. 


\title{
L'immigration en France des enfants nés à l'étranger
}

\author{
Xavier Thierry et Tatiana Eremenko
}

\section{Version}

Libre accès

\section{POUR CITER CETTE VERSION / TO CITE THIS VERSION}

Xavier Thierry et Tatiana Eremenko, 2009, "L’immigration en France des enfants nés à l'étranger",

Recherches familiales: 43-54.

Disponible sur / Availabe at:

http://hdl.handle.net/20.500.12204/AWRIJQ7egpz89Adag6EQ 


\section{L’immigration en France des enfants nés à l'étranger}

\section{Xavier Thierry, Ined}

Tatiana Eremenko, Ined

\section{Introduction}

L'immigration a un impact sur le nombre d'enfants et de couples présents en France. Cet impact se manifeste de deux manières. Il s'opère directement lorsque les immigrés se marient ou ont des enfants sur le territoire national et, indirectement, lorsqu'ils ont vécu ces événements familiaux à l'étranger et migrent ensuite. Les migrations familiales sont représentées par deux catégories de personnes : des conjoints et des enfants. Afin de situer l'ordre de grandeur du phénomène que nous allons examiner, nous proposons de mettre de suite en relation le nombre des migrations familiales d'après ce qu'en disent les statistiques de regroupement familial (effectifs d'enfants et de conjoints ayant bénéficié d'une procédure administrative de regroupement familial) avec les statistiques de naissances et de mariages survenus en France enregistrées en mairie. S'agissant de la formation des couples, signalons qu'il y a davantage d'étrangers mariés à l'étranger arrivant en France pour rejoindre leur conjoint que de couples d'étrangers déjà cohabitant s'y mariant : près de 13000 conjoints étrangers sont venus en France en 2005 dans le cadre d'une procédure de regroupement familial, contre 7000 mariages entre étrangers célébrés en France la même année (ressortissants d'un pays tiers) ${ }^{1}$. Concernant l'histoire migratoire des enfants présents en France, c'est l'inverse qui se produit : la natalité étrangère en France l'emporte nettement sur les migrations d'enfants nés à l'étranger. Près de 50000 naissances de deux parents étrangers (hors Union européenne) ont été enregistrées par l'état civil sur le sol français en 2005, contre seulement un peu plus de 13000 enfants admis par la procédure de regroupement familial. Le flux d'enfants du regroupement familial ne représente donc qu'un nombre très réduit d'enfants comparé à la génération née en France d'au moins un parent immigré. Ceci découle du fait que la plupart des couples se forment à l'étranger, puis mettent au monde la plupart de leurs enfants après s'être installés en France. La majorité des enfants vivant en France avec un

\footnotetext{
${ }^{1}$ En outre, les statistiques de regroupement familial ne restituent qu'une partie des migrations familiales. En effet, un immigré ayant acquis la nationalité française pourra librement faire venir sa famille sans passer par la procédure de regroupement familial. En 2005, les étrangers venus en France grâce à des liens familiaux avec un parent français sont deux fois plus nombreux que les familles d'étrangers du regroupement familial (respectivement 52000 et 26000 , selon les statistiques d'immigration de l'INED). Les enfants issus de ces couples ont la nationalité française à la naissance (du fait de la nationalité de l'un de leur parent); le nombre de ceux venus en France est inconnu.
} 
parent immigré y sont nés. Lorsque les couples mariés à l'étranger ont déjà procréé avant de venir, leur descendance est assez réduite, de l'ordre d'un enfant en moyenne par couple. Le potentiel migratoire que représente les enfants nés à l'étranger dont l'un des parents vit en France est donc en soi assez limité.

Les migrations d'enfants, objet de cet article, constituent un phénomène moins étudié que les migrations conjugales ${ }^{2}$. Il est vrai que la législation sur le regroupement familial s'en est longtemps tenue à durcir les conditions d'arrivée des conjoints pour tenter de d'influer les comportements migratoires et les stratégies familiales. La problématique des enfants a néanmoins surgi dans l'actualité lors du débat sur la loi de novembre 2007 réformant les modalités de regroupement familial concernant les enfants, au travers d'une proposition de test génétique pour prouver la filiation. Depuis 1976, année d'institution de la procédure de regroupement familial, les étrangers en situation régulière en France peuvent se faire rejoindre par un conjoint et/ou des enfants. Qu'entend-on par enfant? Certes la législation mentionne les « enfants du couple, mineurs de 18 ans », mais une définition plus large en est donnée, en particulier depuis la loi du 11 mai 1998, qui précise que le droit au regroupement familial est ouvert aux enfants dont «la filiation n'est établie qu'à l'égard du demandeur ou de son conjoint », ainsi qu'aux enfants adoptés. Un parent divorcé ou les époux d'une famille recomposée sont donc autorisés à faire venir des enfants d'une précédente union. On conçoit mal en effet qu'un parent ayant eu des enfants au cours d'unions successives soit contraint de se séparer d'une partie de ses enfants en laissant au pays d'origine les ainés issus d'une union plus ancienne. Le regroupement familial n'est donc pas réservé aux conjoints ayant vécu une histoire matrimoniale simple. L'une des dispositions de la loi du 21 novembre 2007 introduisant, sous certaines conditions, la possibilité de recourir aux empreintes génétiques afin de déterminer avec certitude la filiation biologique entre l'enfant et la mère semble remettre en cause ce principe de neutralité vis-à-vis de la pluralité des histoires familiales des candidats à l'immigration.

Dans la première section de cet article, on examine dans quelle mesure ces enfants nés à l'étranger appartiennent à des fratries constituées de frères et sœurs nés dans des pays différents. La deuxième section porte sur la fréquence de leur venue en France et la manière dont se succèdent dans le temps les migrations de chacun des parents et de leurs enfants.

\footnotetext{
${ }^{2}$ Les migrations des conjoints ont été analysés dans : X. Thierry, 2007, "Caractéristiques démographiques des ouvrants droit au regroupement familial”, chapitre 4, pp. 89-105 in C. Régnard, Immigration et présence étrangère en France en 2006, Rapport de la Direction de la population et des migrations, La Documentation française.
} 
Enfin la troisième section apporte des éclairages sur les normes parentales relatives à l'âge et au sexe des enfants regroupés.

\section{Sources de données utilisées}

Nous cherchons à apporter un éclairage à l'aide de données statistiques sur les migrations internationales impliquant des enfants étrangers. Au sens large, cela concerne tout enfant né à l'étranger et accompagnant ou venant rejoindre un parent pour vivre en France, que le parent immigré soit étranger ou devenu français au moment de son arrivée. Au sens strict, on peut aussi s'intéresser aux seuls enfants dont les deux parents sont étrangers au moment de la venue de l'enfant et qui doivent suivre les prescriptions de la procédure de regroupement familial. Cette dernière approche est plus restrictive, mais elle s'inscrit davantage dans l'espace d'intervention des pouvoirs publics. Ces deux approches sont illustrées par les deux sources de données utilisées dans cet article.

L'enquête sur l'histoire familiale (EHF), enquête effectuée par l'INSEE et associée au recensement de 1999, a administré un questionnaire de quatre pages à un échantillon de 380000 hommes et femmes d'au moins 18 ans $^{3}$. Les adultes y étaient notamment invités à déclarer l'ensemble des enfants qu'ils ont eus, quel que soit le pays de naissance de ces enfants et leur lieu de résidence au moment de l'enquête (sauf s'ils sont décédés). La descendance ainsi reconstituée précise, pour chaque enfant, son sexe, sa date de naissance, s'il est né en France métropolitaine et s'il y réside actuellement. On connaît également l'année du mariage des personnes enquêtées. En outre, les informations recueillies dans le bulletin de recensement viennent compléter les données de l'enquête en renseignant sur le statut d'immigré des adultes du ménage, leur pays de naissance et l'année d'arrivée en France. Cette dernière information est également disponible pour les enfants résidant au domicile parental.

La réunion de ces informations permet d'embrasser l'ensemble de la descendance des couples d'immigrés, que les enfants soient nés avant ou après la migration des parents, et de replacer ces naissances dans la chronologie migratoire des adultes. Afin de disposer de l'information la plus complète sur l'histoire migratoire du groupe familial, les parents immigrés vivant sans conjoint ne sont pas pris en compte. Seuls ont été retenus les parents tous deux originaires d'un pays tiers (hors Union européenne à 15) afin de ne pas adjoindre

\footnotetext{
${ }^{3}$ C. Lefèvre, A. Filhon, Histoires de famille, histoires familiales, Les Cahiers de l'INED, n 156,641 p.
} 
des situations relevant de la liberté de circulation. La base de données mise en forme pour cette étude est composée de 7500 couples ayant déclaré 28500 enfants.

Les données de cette enquête ne sont pas sans inconvénients. Elles portent sur des familles de migrants dont l'installation en France peut être assez ancienne, et dans tous les cas antérieure à 1999, date de l'enquête. Comme dans toute observation rétrospective, les personnes qui sont reparties ne peuvent plus être interrogées. De plus, les couples divorcés ou séparés ont dû être écartés du champ de l'étude faute d'information sur l'année d'immigration du conjoint avec lequel les enfants ne vivent plus. Autre inconvénient, le sous-échantillon est de taille réduite et le nombre d'observations réellement utilisables est affecté par des non réponses, voire par des erreurs de déclaration, en particulier à la question sur l'année de migration. Enfin, la dimension rétrospective des données empêche de comparer les comportements des migrants arrivés à différentes périodes car plus l'immigration est ancienne, plus les personnes sont par construction arrivées jeunes. L'âge à la migration étant corrélé aux phénomènes familiaux, une telle perspective historique donnerait des résultats biaisés.

Ces inconvénients des statistiques d'enquête disparaissent avec l'emploi de données administratives recueillies en continu. L'INED réalise depuis près de 10 ans des exploitations statistiques du fichier des titres de séjour du ministère de l'intérieur aux fins de comptabilisation des flux d'entrées d'étrangers en France. Ces travaux ont permis plus récemment de produire une première étude statistique sur le regroupement familial ${ }^{4}$. Ces données recensent les demandes de regroupement familial de la période 1993-2005, couvrant 151000 enfants nés à l'étranger. Elles comportent le même type d'informations que dans l'enquête de l'INSEE, notamment l'année de mariage des parents, les années de naissance et de migration pour les personnes appelées. Ces variables sont nécessaires à l'étude de la chronologie du regroupement familial, mais avec l'avantage d'une information obligatoire, non affectée par les biais de non réponses.

\section{Nombre d'enfants nés en France ont au moins un frère ou une sœur nés à l'étranger}

Le pays de naissance en France ou à l'étranger des enfants d'immigrés dépend de l'âge à la migration de leurs parents. Plus longue est la période de vie passée par les parents dans le

\footnotetext{
${ }^{4}$ X. Thierry, 2007, ibid.
} 
pays d'origine avant la migration, plus important sera le nombre d'enfants qu'ils y ont eus. En réalité, une petite fraction seulement des immigrés ont eu tous leurs enfants à l'étranger (11\%), la majorité les ayant tous eu en France (54\%). Néanmoins, très souvent la migration des adultes intervient aux âges de forte fécondité, leurs enfants sont alors pour partie nés à l'étranger, pour partie en France, et appartiennent dans 34\% des cas à des fratries mixtes (tableau 1).

Le pays de naissance des enfants dépend aussi du moment où se sont mariés les parents par rapport à leur histoire migratoire. Les couples qui se sont mariés avant de migrer ont assez souvent des fratries mixtes, à raison de $55 \%$, proportion qui monte à $63 \%$ lorsque les conjoints sont arrivés à des dates différentes. Dans cette catégorie de couples, peu ont donné naissance à tous leurs enfants en France (17\% contre 54\% pour l'ensemble des couples).

Tableau 1: Répartition (\%) des couples selon le pays naissance de leurs enfants et la situation familiale à l'arrivée en France du premier des conjoints

\begin{tabular}{|c|c|c|c|c|c|c|c|}
\hline & \multicolumn{3}{|c|}{$\begin{array}{c}\text { Couples mariés avant la venue des deux } \\
\text { conjoints }\end{array}$} & \multicolumn{3}{|c|}{$\begin{array}{c}\text { Couples mariés après la venue du premier } \\
\text { conjoint }\end{array}$} & \multirow[b]{2}{*}{ Ensemble } \\
\hline & Ensemble & $\begin{array}{c}\text { dont : conjoints } \\
\text { arrivés } \\
\text { ensemble }\end{array}$ & $\begin{array}{l}\text { dont : conjoints } \\
\text { arrivés } \\
\text { séparément }\end{array}$ & Ensemble & $\begin{array}{l}\text { dont : conjoints } \\
\text { mariés en } \\
\text { France }\end{array}$ & $\begin{array}{l}\text { dont : conjoints } \\
\text { mariés à } \\
\text { l'étranger }\end{array}$ & \\
\hline \multicolumn{7}{|c|}{ Répartition du lieu de naissance des enfants d'une même fratrie *: } & \\
\hline $\begin{array}{l}\text { tous les enfants sont nés à } \\
\text { l'étranger }\end{array}$ & 27,1 & 33,0 & 21,9 & 3,4 & 2,6 & 3,9 & 11,4 \\
\hline $\begin{array}{l}\text { les enfants sont nés soit } \\
\text { en France, soit à l'étranger }\end{array}$ & 55,4 & 47,3 & 62,6 & 23,4 & 12,4 & 31,4 & 34,2 \\
\hline $\begin{array}{l}\text { tous les enfants sont nés } \\
\text { en France }\end{array}$ & 17,5 & 19,7 & 15,5 & 73,2 & 85,0 & 64,7 & 54,4 \\
\hline Total & 100 & 100 & 100 & 100 & 100 & 100 & 100 \\
\hline Répartition des couples & 34,9 & 16,7 & 18,2 & 65,1 & 25,5 & 39,6 & 100,0 \\
\hline $\begin{array}{l}\text { * enfants issus de parents ré } \\
\text { Champ : couples d'immigrés } \\
\text { Source: INSEE, Etude de l'h }\end{array}$ & $\begin{array}{l}\text { sident en Fra } \\
\text { ressortissan } \\
\text { istoire familia }\end{array}$ & $\begin{array}{l}\text { ance depuis au m } \\
\text { tts d'un pays tiers } \\
\text { ale (EHF), } 1999 .\end{array}$ & $\begin{array}{l}\text { loins } 5 \text { ans } \\
\text { à l'Union europé }\end{array}$ & nne (à 15) & & & \\
\hline
\end{tabular}

Toutefois, les couples ayant débuté leur vie commune à l'étranger sont minoritaires (35\%) parmi les couples d'immigrés présents en France. Deux couples d'immigrés sur trois se sont mariés après la venue en France de l'un des conjoints, et on peut aussi distinguer ceux qui se sont mariés en France (25\%) de ceux, les plus nombreux, qui se sont mariés à l'étranger $(40 \%)$.

La part des enfants nés à l'étranger y est nécessairement assez faible puisqu'a priori ces conjoints ont vécu peu de temps ensemble à l'étranger. En effet, 15\% des couples mariés en France ont eu au moins un enfant à l'étranger, contre plus de $80 \%$ pour les couples qui se sont 
mariés avant de quitter le pays. Les couples mariés à l'étranger alors que l'un des conjoints vivait déjà en France ont assez souvent eu leurs enfants à l'étranger (35\%), alors que leur mariage a consisté en une union à distance, les fréquentations étant circonscrites à des retrouvailles temporaires, aussi longtemps que le deuxième conjoint n'a pas définitivement migré. Leur relation a pu durer un certain nombre d'années avant que ne débute la vie commune en France. Il est possible qu'en se mariant, les conjoints escomptaient vivre ensemble rapidement, mais que ce projet ait été différé pour différentes raisons (par exemple à défaut de remplir les conditions légales du regroupement familial ${ }^{5}$ ). Pour l'immigré résidant en France, la présence à l'étranger d'un conjoint avec lequel on s'est marié avant de partir ou qu'on est allé épouser alors que l'on résidait déjà en France n'empêche donc pas la poursuite ou le commencement de la vie féconde. Ce caractère transnational des familles immigrées se manifeste au travers de deux éléments liés à leurs enfants: l'existence de fratries mixtes du point de vue du pays de naissance de chaque enfant composant la fratrie et la disjonction entre le pays de naissance des enfants et le pays de résidence de l'un des parents au moment de la naissance des enfants.

\section{La migration de la mère polarise la venue de ses enfants}

Les enfants nés à l'étranger forment une part réduite de la descendance des couples d'immigrés qui ont la plupart de leurs enfants en France. Il est cependant intéressant d'étudier dans quelles conditions ces enfants viennent en France.

La plupart des couples interrogés ont fait venir leurs enfants nés à l'étranger : près de $92 \%$ de ces derniers sont déclarés comme vivant en France, contre 8,5\% qui sont restés (tableau 2). Cet indicateur mesure approximativement la fréquence du regroupement familial au bénéfice d'enfants mineurs, à la réserve près que les enfants résidant à l'étranger ont pu venir en France, puis repartir une fois adulte. A l'inverse, il est vraisemblable qu'une fraction des enfants nés à l'étranger ait été omise, les parents déclarant avec moins de précision les enfants avec lesquels ils n'ont jamais vécu en France. Ce dernier facteur explique sans doute une fréquence de regroupement familial aussi proche de 100\%, même si la constitution d'un échantillon de couples d'immigrés conduit à en prendre la mesure dans la population la plus encline à l'unité familiale. Des recherches antérieures donnent des mesures plus faibles du regroupement familial, descendant jusqu'à $50 \%$ dans certains courants ${ }^{6}$. Parmi les différents courants étudiés dans l'enquête MGIS en 1992, deux types de pays ont été distingués : d'une

\footnotetext{
${ }^{5}$ Disposer de ressources financières suffisantes et d'un logement de taille adapté au nombre de personnes.

${ }^{6}$ M. Tribalat : Enquête Mobilité géographique et insertion sociale de 1992, rapport final, 1993, 555 pages.
} 
part, le Maroc et l'Algérie où le regroupement est tardif et incomplet, d'autre part la Turquie où il est tardif mais complet. Il est aussi plus fréquent chez les migrants venus célibataires que chez les migrants venus mariés.

Tableau 2 : Proportion (\%) d'enfants nés à l'étranger et y vivant encore au moment de

l'enquête

\begin{tabular}{|c|c|c|c|c|}
\hline & \multirow[b]{2}{*}{$\begin{array}{c}\% \text { des enfants } \\
\text { vivant à l'étranger }\end{array}$} & \multicolumn{3}{|c|}{ Perspective migratoire en France : } \\
\hline & & $\begin{array}{l}\text { encore } \\
\text { susceptible de } \\
\text { migrer }\end{array}$ & $\begin{array}{l}\text { individu } \\
\text { maintenant } \\
\text { majeur }\end{array}$ & $\begin{array}{l}\text { individu déjà } \\
\text { majeur lors de la } \\
\text { migration du } \\
\text { dernier migrant }\end{array}$ \\
\hline $\begin{array}{l}\text { tous les frères et sœurs sont nés à } \\
\text { l'étranger }\end{array}$ & 14,1 & 2,1 & 6,3 & 5,7 \\
\hline $\begin{array}{l}\text { les enfants sont nés soit en France, } \\
\text { soit à l'étranger }\end{array}$ & 6,0 & 1,2 & 3,4 & 1,4 \\
\hline \multicolumn{5}{|l|}{ Pays du père } \\
\hline Algérie & 10,4 & 0,8 & 7,0 & 2,6 \\
\hline Maroc & 6,1 & 0,6 & 2,5 & 3,0 \\
\hline Tunisie & 3,6 & 0,1 & 2,1 & 1,4 \\
\hline Afrique subsaharienne & 11,2 & 6,4 & 3,5 & 1,3 \\
\hline Turquie & 4,1 & 0,9 & 2,8 & 0,4 \\
\hline les parents sont arrivés ensemble & 8,1 & 0,6 & 3,7 & 3,8 \\
\hline les parents sont arrivés séparément & 8,5 & 1,7 & 3,7 & 3,1 \\
\hline Enfant de sexe masculin & 8,0 & 1,3 & 4 & 2,7 \\
\hline Enfant de sexe féminin & 9,1 & 1,7 & 4,6 & 2,8 \\
\hline Ensemble & 8,5 & 1,5 & 4,3 & 2,7 \\
\hline \multicolumn{5}{|c|}{$\begin{array}{l}\text { Champ : couples d'immigrés ressortissants d'un pays tiers à l'Union européenne (à 15) ayant eu } \\
\text { au moins un enfant à l'étranger. } \\
\text { Source: INSEE, Etude de l'histoire familiale (EHF), } 1999 \text {. }\end{array}$} \\
\hline
\end{tabular}

Parmi ces couples ayant eu des enfants à l'étranger, très peu d'enfants sont encore susceptibles de venir en France avant 18 ans (1,5\%). La plupart des enfants vivant à l'étranger sont maintenant majeurs ou l'étaient déjà lors de la migration des parents (respectivement $4,3 \%$ et $2,7 \%$ ). Les parents ayant mis au monde des enfants à la fois en France et à l'étranger ont moins souvent laissé leurs enfants à l'étranger (6\%), comparé à ceux qui ont mis au monde tous leurs enfants à l'étranger (14\%). L'histoire de ces deux types de fratrie a peut-être été assez différente : les fratries d'enfants tous nés à l'étranger sont issus de parents ayant migré à un âge plus élevé et dont le projet d'installation à l'étranger a été pensé après la naissance des enfants. A l'inverse, lorsque la fratrie est mixte, on peut supposer que le projet familial et migratoire constituent un projet de vie plus global et qu'il y a une volonté des parents de réunir les frères et sœurs par delà les frontières séparant leur naissance. On observe 
dans le premier cas que 5,7\% des enfants ont déjà dépassé la majorité au moment de la migration des parents, contre seulement $1,4 \%$ dans le second cas. Les fratries d'enfants composées exclusivement de natifs du pays d'origine ont donc sans doute plus fréquemment grandi auprès des proches de la famille à l'étranger et y seraient plus souvent restées. Plus de $10 \%$ des enfants nés à l'étranger de parents algériens ou subsahariens vivent à l'étranger, mais les situations sont différentes compte tenu de leur inégale ancienneté en France : les enfants d'Algériens ont souvent désormais dépassé l'âge de la majorité et la fréquence des regroupements familiaux passés y est peut être sous-estimée (compte tenu des retours en tant qu'adulte), tandis que les enfants des pays d'Afrique noire, d'implantation plus récente, sont encore suffisamment jeunes pour laisser ouverte la possibilité d'un regroupement familial dans les années à venir.

Seulement un quart des enfants ont déclaré être arrivés avec leurs parents, c'est-à-dire la même année (tableau 3). La plupart du temps (70\%), l'homme arrive en premier, puis est rejoint par sa femme qui, huit fois sur dix (78\%), vient avec ses enfants. Il est assez rare que les enfants arrivent seuls, après la migration des parents ou en intercalé. Il accompagne très souvent au moins l'un des parents : 56,4\% la mère seulement, $23,7 \%$ les deux parents, $1,8 \%$ le père seulement, soit au total $82 \%$ des enfants accompagnent au moins l'un des parents. Quand leur migration s'effectue une année différente de celle des parents, la situation la plus courante est celle d'enfants arrivés après l'installation du couple (14\%), tout se passant comme si l'accueil des enfants nécessitait des préparatifs qu'un couple réuni est le plus apte à mettre au point. Il est plus rare que des enfants rejoignent un seul de leurs parents alors que le deuxième ne viendra que plus tard (3\%). Le choix du moment opportun pour faire venir ses enfants est très lié à celui de l'arrivée de la mère : qu'elle arrive après son mari ou le devance, sa migration polarise celle de ses enfants.

Tableau 3 : Moment d'arrivée des enfants nés à l'étranger selon l'ordre de migration des parents 


\begin{tabular}{|l|c|c|c|c|}
\hline L'enfant est arrivé : & $\begin{array}{c}\text { parents arrivés } \\
\text { ensemble }\end{array}$ & $\begin{array}{c}\text { père arrivé } \\
\text { avant la mère }\end{array}$ & $\begin{array}{c}\text { mère arrivée } \\
\text { avant le père }\end{array}$ & Ensemble \\
\hline avec les deux parents & 90,4 & - & - & 23,7 \\
\hline avec le père & - & 1,2 & 27,5 & 1,8 \\
\hline avec la mère & - & 78,1 & 40,4 & 56,4 \\
\hline entre les migrations des parents & - & 4,2 & 6,9 & 3,2 \\
\hline après la migration des parents & 8,1 & 16,3 & 22,9 & 14,3 \\
\hline avant la migration des parents & 1,5 & 0,2 & 2,3 & 0,6 \\
\hline Total & 100,0 & 100,0 & 100,0 & 100,0 \\
\hline
\end{tabular}

De même que les migrations adultes sont fréquemment espacées dans le temps, il arrive également que la venue du groupe d'enfants nés à l'étranger s'effectue de façon échelonnée. On a donc calculé pour les fratries d'au moins deux enfants nés à l'étranger, la proportion d'entre eux arrivés la même année ou une année différente. Les frères et sœurs arrivent très souvent tous ensemble, c'est-à-dire huit fois sur dix. La dissociation des fratries à un moment de leur enfance du fait de la migration est nettement moins fréquence que dans l'histoire des couples, où inversement l'arrivée en France à des dates différentes se produit dans la majorité des cas. On observe aussi que l'échelonnement des migrations d'adultes a pour corollaire l'espacement de celles de leurs enfants : les parents arrivés ensemble font plus rarement venir leurs enfants séparément les uns des autres (9\%) que les parents arrivés séparément (27\%).

\section{Les enfants arrivent de plus en plus âgés}

Les enfants sont de plus en plus âgés au moment de leur arrivée en France : $31 \%$ des enfants regroupés en 1990 avaient moins de 6 ans, cette proportion n'étant plus que de 25\% en 1995, de $20 \%$ en 2000, de $18 \%$ en 2005 (source ANAEM). Autrement dit, selon les informations disponibles au ministère de l'intérieur (source AGDREF), la moitié des enfants ayant fait l'objet d'une demande de regroupement familial ont moins 10 ans en 2005. Cet âge médian varie selon la situation familiale au moment de l'arrivée du premier des parents (figure 1). Il est nécessairement plus élevé lorsque le parent est venu marié, laissant au pays des enfants qu'il a eu avant de partir et ayant déjà atteint un certain âge. Ces derniers ont connu le départ de l'un de leur parent vers l'âge de 6 ans. De surcroit, les parents ne les font 
pas venir immédiatement : le délai minimum légal pour regrouper sa famille est désormais fixé à deux ans, mais en réalité, le temps passé en France est voisin de 7 ans. Au total, cette catégories d'enfants est celle dont l'âge à l'arrivée est le plus tardif, s'établissant à plus de 12 ans en 2005 .

Figure 1: Age des enfants au dépôt de la demande de regroupement familial selon la période et le type de migrant

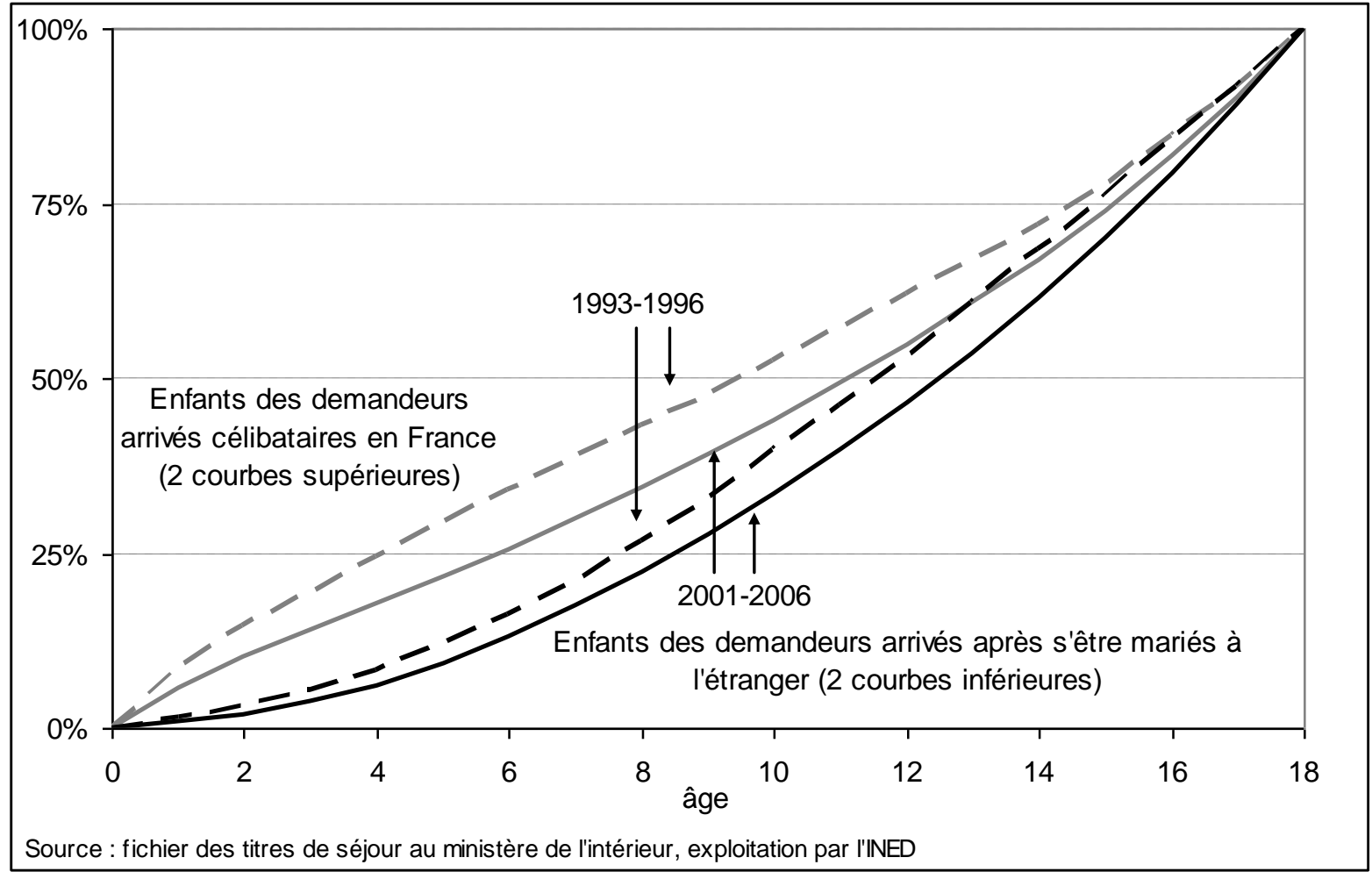

Note de lecture : La moitié des enfants dont les parents ont effectué une demande de regroupement familial en 2001-2006 sont âgés d'environ 11 ans lorsque le premier parent arrivé en France est venu célibataire, 12,5 ans s'il est arrivé déjà marié.

Lorsque les enfants sont nés après le départ du premier des parents, ils sont immédiatement aptes à le rejoindre étant donné que ce dernier satisfait déjà largement au critère légal de durée de séjour. Les migrants adultes arrivés célibataires et s'étant mariés au pays d'origine ont eu leurs enfants en moyenne 14 ans après le début de leur séjour en France. La naissance de leurs enfants aurait pu être l'occasion de mettre un terme à la dispersion géographique de la famille. Tel n'est pas du tout le cas puisque la moitié des enfants arrivés en 2005 avaient plus de 11 ans, un âge médian à l'arrivée finalement peu éloigné de celui des enfants nés avant la migration des parents (12 ans, cf. supra). Ces arrivées tardives sont en partie dues aux difficultés rencontrées par les parents pour obtenir de l'administration l'autorisation de regroupement familial, la fréquence des demandes ajournées par l'administration étant 
d'environ 30\%. L' « intérêt des enfants », du point de vue de la société d'accueil, serait plutôt en faveur d'une arrivée précoce en France afin d'y mener une vie familiale recomposée et y recevoir une scolarisation la plus complète possible. Néanmoins d'autres raisons inclinant les parents à faire venir leurs enfants assez âgés semblent prégnantes, d'autant que la prise en charge de ces enfants dans leur pays d'origine est souvent réglée par la présence de la mère.

\section{Une préférence pour la venue des garçons}

L'analyse des chiffres de l'immigration des enfants du regroupement familial fait apparaître que les parents font venir leurs filles à des âges légèrement plus jeunes que les garçons. Mais ce léger report ne doit pas faire croire que les filles ont plus de chances de venir vivre avec leurs deux parents en France. Au contraire, la répartition des enfants du regroupement familial penche en moyenne en faveur des garçons, significativement au-dessus du niveau de surmasculinité à la naissance. Les garçons représentent $54 \%$ des effectifs d'enfants, contre $46 \%$ de filles (tableau 4). Cette sous-représentation des filles s'accroît à mesure que l'âge s'élève, les enfants ayant plus de 16 ans au moment du regroupement familial étant seulement à $40 \%$ de sexe féminin. Probablement l'introduction préférentielle par le canal du regroupement familial de garçons hors de l'obligation scolaire est-il utilisé pour les faire entrer sur le marché du travail. Il se peut aussi que les parents préfèrent que leurs filles restent au pays d'origine afin de s'y marier, selon les intérêts et désirs de la famille ou du clan. Ce qu'une immersion dans la société française en tant que célibataire, avec l'émancipation en corolaire, pourrait contrarier.

Toutefois, cette préférence pour les garçons disparaît si les enfants rejoignent leur mère déjà installée en France (51\% sont des filles). Elle est en revanche particulièrement accentuée lorsque le seul parent installé en France est le père (44\%), et plus encore lorsque les enfants ne sont pas accompagnés de leur mère. Tout se passerait donc comme si l'arrivée des enfants de chaque sexe était aussi déterminée par des représentations liées au genre, les mères étant le pivot de la prise en charge des enfants, surtout s'agissant de l'éducation des filles. Ces indicateurs ne différent guère d'un pays à l'autre, ce qui semble écarter l'idée selon laquelle cette dimension sexuée du regroupement familial s'enracinerait dans des cultures spécifiques à quelques pays. Il semble que ce soit plutôt l'expression d'une stratégie générale des migrants. 
Tableau 4 : Proportions (\%) de filles parmi les enfants du regroupement familial selon le sexe du parent soumettant la demande

\begin{tabular}{|c|c|c|c|}
\hline & $\begin{array}{c}\text { père arrivé avant la } \\
\text { mère }\end{array}$ & $\begin{array}{l}\text { mère arrivée avant le } \\
\text { père }\end{array}$ & Ensemble \\
\hline \multicolumn{4}{|c|}{ Age des enfants au moment de la demande de regroupement familial } \\
\hline $0-5$ ans & 48 & 53 & 48 \\
\hline $6-12$ ans & 47 & 51 & 48 \\
\hline $13-15$ ans & 42 & 52 & 45 \\
\hline $16-17$ ans & 38 & 49 & 40 \\
\hline \multicolumn{4}{|c|}{ Etat matrimonial du parent demandeur au moment de son arrivée } \\
\hline célibataire & 44 & 53 & 44 \\
\hline marié & 45 & 51 & 47 \\
\hline \multicolumn{4}{|c|}{ Pays d'origine de la famille } \\
\hline Turquie & 45 & 47 & 45 \\
\hline Afrique subsaharienne & 44 & 52 & 47 \\
\hline Maroc & 43 & 50 & 44 \\
\hline Tunisie & 42 & 50 & 43 \\
\hline Algérie & 46 & 52 & 48 \\
\hline Ensemble & 44 & 51 & 46 \\
\hline \multicolumn{4}{|c|}{ Champ : enfants ayant fait l'objet d'une demande de regroupement familial entre 1993 et 2005} \\
\hline
\end{tabular}

\section{Conclusion}

La société française a vivement débattu à l'automne 2007 d'une proposition législative de recourir à des tests génétiques entre la mère et l'enfant né à l'étranger, lors de l'examen des demandes de regroupement familial. Se sont trouvées alors posées des interrogations sur la nature des structures familiales dans les sociétés non occidentales et sur les stratégies des migrants pour contourner les obstacles à leur installation en Europe.

Que représentent statistiquement ces situations familiales complexes de nature à faire naître des doutes sur l'authenticité des liens de parenté biologiques ? Est-il vraisemblable que des enfants soient présentés comme les siens, voire formellement adoptés, dans un but migratoire ? Cet article montre la part assez modeste des enfants nés à l'étranger dans la descendance des couples d'immigrés, l'essentiel de leurs enfants naissant en France. Certaines conjonctions familiales assez improbables font néanmoins planer quelques doutes sur la réalité de certaines filiations biologiques et sur la possibilité qu'une fraction des enfants nés à 
l'étranger aient été confiés à des adultes de l'entourage susceptibles de les faire migrer en France. En effet, un tiers d'entre eux ont vu le jour alors que l'un des parents présumé vivait en France, un cinquième des enfants sont venus en France à une date différente des autres membres de leur fratrie ou encore un dixième sont nés après que les parents aient eu des enfants en France.

\section{Résumé}

Utilisant des données récentes (enquête Etude de l'Histoire Familiale de l'Insee et registre administratif des étrangers), l'article traite du déroulement du parcours génésique et migratoire des couples d'immigrés résidant en France.

L'ordre des deux événements (migrations et mariage des conjoints) a un impact sur le pays de naissance des fratries d'enfants, intervenant exclusivement en France ou à l'étranger, ou bien dans l'un et l'autre des espaces. Un tiers des parents ont eu des enfants dans deux contextes nationaux différents.

Les enfants nés à l'étranger arrivent de plus en plus âgés, après 10 ans, et la venue des garçons est préférée à celle des filles. Leur venue est souvent concomitante à celle de leur parents : trois fois sur quatre, ils sont arrivés en même temps que leur mère. 